\title{
Apuntes sobre la contribución del GAT a la Atención Temprana
}

\section{Notes on the contribution of GAT for Early Intervention}

\section{Palabras clave:}

Atención Temprana, asociación, trastornos en el desarrollo, niños/ as, familias, Centros de Desarrollo Infantil y Atención Temprana.

\section{Keywords:}

Early Intervention, association, developmental disorders, children, families, Childcare Centers and Early Intervention.

\section{Introducción}

La Atención Temprana (en adelante, AT) a los niños y niñas con dificultades en su desarrollo o con riesgo de padecerlas y a sus respectivas familias, ha experimentado en nuestro país un desarrollo tan espectacular que la ha conducido desde la casi inexistencia a una madurez encomiable en muy pocas décadas. Sin temor a equivocarnos, podemos afirmar con satisfacción que hoy cualquier niño o niña con discapacidad o riesgo de padecerla y sus familias, tienen reconocido el derecho a ser atendidos por los servicios públicos tan pronto es detectada su situación.

En el presente artículo pretendo resaltar la contribución -modesta pero eficaz- del Grupo de Atención Temprana (GAT) a la reflexión, análisis de la situación, debate interprofesional e interterritorial en torno a la AT, por un lado, al reconocimiento de la importancia de la AT en el desarrollo de los niños y niñas que la necesitan por parte de la sociedad y sus representantes políticos y, finalmente, a la implantación de la AT en la totalidad del territorio nacional.

Con este objetivo, propongo un breve recorrido por las circunstancias

\section{Fátima Pegenaute Lebrero $<$ fpegenaute@bcn.cat>}

Psicóloga del Equip d'Atenció Precoç, EIPI, de Nou Barris. IMD de Barcelona. Ex presidenta de la Federación Estatal de Profesionales de la Atención Temprana
Para citar:

Pegenaute, F. (20I6): “Apuntes sobre la contribución del GAT a la Atención Temprana”, Revista Española de Discapacidad, 4 (I): 225-239.

Doi: <http://dx.doi.org/I0.5569/23405IO4.04.OI.I3> 
que llevaron a los profesionales de la AT a agruparse en torno a iniciativas de formación, debate y reflexión sobre su experiencia pionera. Experiencias que, casi siempre, nacían alrededor de hospitales de niños, servicios de neonatología, escuelas y asociaciones relacionadas con la discapacidad. De la necesidad de formalizar y compartir las reflexiones sobre la problemática de la AT nacieron las primeras asociaciones de profesionales.

En ese contexto surgió el GAT, cuyos miembros compartieron identidad y denominación con la Federación Estatal de Asociaciones de Profesionales de la Atención Temprana. En adelante, GAT y Federación serán términos casi sinónimos en el argot de la AT. El esbozo rápido -casi una crónica- del proceso de nacimiento, constitución y aportaciones a la AT de este grupo de profesionales quiere ser una expresión de reconocimiento y agradecimiento a cuantas instituciones y personas contribuyeron y siguen contribuyendo a la fecunda realidad que es hoy la AT en nuestro país. Los niños y niñas con dificultades en su desarrollo y sus familias son los principales beneficiarios.

\section{El asociacionismo en la Atención Temprana}

El nacimiento de las asociaciones de profesionales involucrados en la AT en España surge, lógicamente, con el despertar del interés -a partir de la conciencia de su necesidad- y el crecimiento de servicios en torno a la atención de los niños con problemas en el desarrollo y sus familias. La complejidad de estos servicios atendidos por profesionales de diversas disciplinas y procedentes de diferentes ámbitos (social, sanitario y educativo), lo novedoso del trabajo en sí y las diferencias en criterios de actuación animó a unos cuantos profesionales en algunas comunidades autónomas a la creación de asociaciones de Atención Temprana. Este es el caso de las primeras, en Cataluña y Valencia. Luego fueron apareciendo muchas otras.
En Cataluña, ya en el año I984, se había creado una Coordinadora d'Equips d'Atenció Precoç que se reunían periódicamente en la sede de la Federació pro Persones amb Discapacitat Intelectual (APPS). Estas reuniones tenían como objetivos poner en común la forma de trabajo de cada equipo, discutir qué modelo de actuación sería el más adecuado y plantear la necesidad de conectarse con la Administración Catalana, con el fin de consensuar las bases de lo que tendría que ser la AT en su territorio. Algunos años después este grupo se formalizó en lo que sería l'Associació Catalana d'Atenció Precoç.

Casi paralelamente, otras entidades y en otros lugares, se unieron para formar grupos de trabajo. Nos referimos a GENMA (Grupo de Estudios Neonatales) y al grupo PADI (Prevención y Atención al Desarrollo Infantil), ambos de Madrid (I987).

El grupo PADI, integrado por profesionales de la salud, de la educación y los servicios sociales, se creó como iniciativa en torno a la prevención y la atención temprana de las discapacidades de los niños y sus familias. Sus principales promotores fueron Felipe Retortillo y José Arizcun. A su vez, Arizcun, en el año I99I, creó GENYSI (Grupo de Estudios Neonatales y Servicios de Intervención) que contó con una red de información muy completa de todo cuanto se realizaba en el Estado en relación al cuidado y tratamiento de los niños pequeños con problemas en el desarrollo o con riesgo de tenerlos. La organización GENYSI, con el apoyo del Real Patronato sobre Discapacidad, inició la celebración de unas reuniones interdisciplinarias anuales, a las que comenzamos a asistir con asiduidad muchos profesionales de la AT de las diferentes comunidades autónomas.

En general, la situación de la Atención Temprana de los niños con discapacidad antes de los años 80 , se abordaba desde una perspectiva asistencial, no sectorial y de tipo médico-sanitario, ya que los equipos existentes estaban ubicados, principalmente, en centros hospitalarios.

El planteamiento de estos modelos, que primaban aspectos parciales en la atención al 
niño con trastornos del desarrollo, se resentían, a nuestro entender, de estos aspectos:

I. Prevención: Prioritariamente y, casi en exclusiva, se primaba la prevención desde el punto de vista biológico (detección de metabolopatías, campañas de vacunación, mejora en la atención perinatal, etc.) sin contemplar los aspectos más psicosociales que también tienen una incidencia tan o más considerable en la génesis de las disfunciones.

2. Detección y diagnóstico: La detección quedaba limitada a los pocos programas hospitalarios y de seguimiento de niños con riesgo biológico y al diagnóstico de los niños con patologías instauradas. El diagnóstico se basaba en considerar básicamente los aspectos biológicos del déficit, dada la formación fundamentalmente médica de los profesionales a los que se les confiaba este trabajo, ignorándose casi totalmente el concepto de riesgo social y de un diagnóstico global.

Muchos niños llegaban a los servicios de diagnóstico a edades ya avanzadas $(4,5$ y 6 años) mayoritariamente procedentes de las escuelas, sin haber recibido en ningún momento, ellos y sus familias, ningún tipo de atención psicológica. En muchos casos, la situación de estos niños, tanto a nivel cognitivo como personal, había empeorado y las familias, sin recibir ningún tipo de información ni soporte, tenían dificultades reales en la relación y manejo de sus hijos.

3. La intervención terapéutica: El inicio de la intervención se realizaba excesivamente tarde o no se hacía. Las intervenciones que se ofrecían como "Estimulación o rehabilitación Precoz" ponían en general su énfasis en una serie de actuaciones que tenían como objetivo, casi de forma exclusiva, al niño enfermo y sus déficits, contemplando a veces a la familia desde una perspectiva lejana.
Ya en I973, un equipo pionero en la Estimulación Precoz en Argentina -me refiero al de Lydia Coriat- detectaba estos mismos problemas y se formulaba la pregunta sobre la eficacia de este tipo de terapias que, de forma simultánea, se dirigían únicamente a los niños según la gravedad y la diversidad de los trastornos que se manifestaban.

Analizaban así la situación: el profesional o profesionales responsables de la intervención diseñaban, de forma individual y cada uno desde su especialidad, un programa o programas diversos, programaban ejercicios dirigidos exclusivamente a corregir o paliar las discapacidades, no pudiendo valorar los intereses del niño, su estado emocional ni las características de su entorno. El profesional instruía luego a los padres para que éstos pudieran proseguir en casa el trabajo iniciado, valorando muy poco las posibles competencias, motivaciones o dificultades emocionales de los propios padres.

En esta organización, la familia tomaba lógicamente un papel subsidiario y sumiso respecto al protagonismo y al saber ejercido por el profesional o profesionales especializados, que organizaban e instruían, cada uno desde su discurso, a los padres.

Así, pues, el inicio exacto del interés por la entonces llamada Estimulación Precoz, en nuestro país, es impreciso y realmente haría falta poder recopilar hechos y actuaciones, que tuvieron su importancia en cada una de las autonomías. Sí que queremos resaltar que fue una consecuencia lógica de la llegada a nuestro país de nuevas teorías y actuaciones procedentes particularmente de los Estados Unidos. Estas aportaciones, basadas en investigaciones sobre la plasticidad del cerebro - particularmente en los primeros años de vida-, los nuevos conceptos de la neurología evolutiva y la psicología de la conducta y del desarrollo, se dirigían hacia la infancia con discapacidad. Todos estos trabajos tenían en común resaltar los efectos positivos y el reconocimiento de que las expectativas de mejora eran resultado, sobre todo, de una 
temprana detección, un diagnóstico y una temprana intervención en estos niños.

Entre los años I980 y I990 comienzan a surgir en muchas comunidades autónomas los primeros centros de intervención llamados, como se ha dicho, de Estimulación Precoz. Estos centros nacieron desde el interés y la iniciativa, sobre todo, del ámbito privado, es decir desde algunas de las asociaciones de padres con niños con diferentes discapacidades y también desde algunos centros hospitalarios.

Ente el I985 y I987 se crean los Equipos multiprofesionales de Atención Temprana -con esta denominación- en todas las provincias del llamado "territorio MEC", a fin de dar cobertura a la atención de alumnos de o a 6 años, al amparo de la LOGSE, que atendían escuelas infantiles. En la Comunidad de Madrid, por ejemplo, constaban de médico, psicólogos, pedagogos, logopedas, maestros especialistas en Pedagogía Terapéutica y trabajadores sociales. En otras comunidades estaban formados por psicólogos, pedagogos, logopedas y trabajadores sociales. Constituían una respuesta a las necesidades de estos niños desde el ámbito educativo. Muchos de estos equipos todavía siguen funcionando en la actualidad. Creo que fueron de los primeros en adoptar la nomenclatura de Atención Temprana.

Sin pretender profundizar en el tema de la normativa legal, convendrá mencionar que las primeras legislaciones surgieron también entre los años 80 y 90. Eran leyes más ligadas a la oferta de diferentes prestaciones desde el ámbito sanitario que al establecimiento de servicios de AT. Quizás la primera de estas leyes es la que se constituyó en Cataluña en el año I983 en materia de asistencia y servicios sociales que establecía los servicios de atención precoz dentro de la atención especializada a personas con disminución.

\section{El grupo de trabajo de Atención Temprana (GAT)}

Debió ser en I995, en el marco de una de las reuniones interdisciplinares -a las que se ha hecho referencia-, cuando entre bastidores y en algún momento de descanso, algunos profesionales procedentes de diferentes comunidades autónomas nos pusimos a compartir, cada uno desde su propia perspectiva, cómo estaba organizada la Atención Temprana en su respectivo lugar geográfico.

Rápidamente se pudo constatar que las diferencias en la organización de los servicios de AT entre las autonomías eran muchas. Los recursos existentes, los criterios que determinaban y regulaban la posibilidad de recibir las prestaciones y la forma de estructurar los servicios en cada uno de los territorios eran muy diversos. Esta diversidad posiblemente se originaba, sobre todo, por los distintos niveles de competencias transferidas desde la Administración Central a las Administraciones Autonómicas. Mientras que unas poseían ya una red de centros consolidada, otras todavía no disponían de una infraestructura básica. Estas grandes diferencias suponían que un niño y su familia no recibían la misma atención ni tenían los mismos recursos en un lugar o en otro.

A partir de este encuentro, surgió el compromiso de seguir profundizando en el tema y estudiar la manera de poder avanzar en él. Había que defender que el mapa de recursos en AT debía armonizar las diferencias territoriales y los diferentes modelos de funcionamiento. Es a partir de estas ideas que se promueve una convocatoria, esta vez de manera formal, que tuvo lugar en la ciudad de Zaragoza en torno al mes de junio del año I996.

La iniciativa de este encuentro partió de la Associació Catalana d'Atenció Precoç, la Asociación Valenciana y de diferentes servicios de Atención Temprana de Aragón y Murcia. Esta reunión tuvo lugar en la sede de la Fundación Rey Ardid, siendo su presidente Jesús 
Sebastián. En ella se propusieron algunos temas como la elaboración de un documento que intentara unificar criterios de tipo conceptual y organizativo a nivel estatal y que pudiera defender el reconocimiento al derecho de recibir AT para toda la población infantil que lo pudiera necesitar.

En el mes de enero de 1997, se convocaba la segunda reunión, ahora ya entre representantes de diferentes autonomías, asistiendo a ella un profesional de la Asociación de Valencia (Juan Carlos Belda), un representante de FEAPS (Federación de Asociaciones a favor de Personas con Disminución Intelectual) de Madrid (Julio Muñoz), una psicóloga de un equipo de AT del País Vasco (Carmen Maza) y dos personas representantes de l'Associació Catalana d'Atenció Precoç (Concha Bugié y Fátima Pegenaute). En este encuentro se va madurando la forma y manera de iniciar un trabajo de análisis y discusión de las diferentes realidades existentes. Igualmente, se hicieron proyectos para poder informar a otros profesionales, entidades y asociaciones del resto de España para que, si lo valoraban de interés, se uniesen a este trabajo.

En abril del mismo año se realizó en Barcelona una tercera reunión a la que se incorporaría $\mathrm{M}^{\mathrm{a}}$ Gracia Milla, presidenta en aquel momento de la Asociación de Atención Temprana de Valencia, que después de haber participado en la realización de muchos de los documentos elaborados por el grupo GAT, se convertiría años más tarde en la primera presidenta del GAT. A esa reunión también se incorporaron otros profesionales de la Asociación de Murcia (Isabel Casbas) y de la Asociación de Galicia (Jaime Ponte).

En estas convocatorias, además de poder intercambiar informaciones contrastadas sobre el funcionamiento de la AT en cada comunidad, se comenzó a debatir sobre algunos conceptos y definiciones utilizados hasta el momento y a esbozar el esquema y los temas principales que serían susceptibles de ser elaborados y recogidos en un documento que más tarde y con el tiempo llegaría a ser el "Libro Blanco de la Atención Temprana”.

\section{La contribución del GAT a la Atención Temprana}

Sin querer ser exhaustivos, se recogen aquí las aportaciones que consideramos más significativas del GAT.

\subsection{Elaboración del Libro Blanco de la Atención Temprana}

El Libro Blanco (GAT, 2000) -su planteamiento, debate, edición y difusión por todo el territorio nacional- fue, sin duda, la gran aportación del GAT y la obra que más contribuyó tanto al prestigio de los miembros del grupo como a la misma AT. En estas líneas, además de recoger algunas fases de la elaboración del proyecto, deseo dejar constancia de la importante contribución del Real Patronato sobre Discapacidad al desarrollo del trabajo del GAT.

Los primeros objetivos que se consensuaron para acometer la elaboración del documento fueron:

I. Elaborar un mapa que objetivara la problemática específica de la AT y sirviera para establecer una adecuada planificación en todo el territorio estatal.

2. Justificar la necesidad de este tipo de intervención para todos los niños que la pudieran necesitar y no únicamente aquellos que tuvieran el "Certificado de discapacidad" -entonces conocido como de disminución o minusvalía, según los territorios-.

3. Proponer una forma coordinada e interdisciplinar de colaboración entre profesionales de distintas disciplinas y ámbitos de trabajo.

4. Establecer unas bases de carácter normativo, abiertas a las características y a la historia de cada territorio. 
En junio y septiembre de I997 se realizaron otras reuniones, esta vez en Valencia -en la sede del Colegio de Psicólogos- y Murcia -en el Departamento de Psicología de la Universidad-. A estas reuniones se incorporó una profesional procedente de Asturias, de la plantilla de la ONCE (Rosa Mayo).

Durante estos encuentros, cada nuevo concepto introducido requería de muchas horas de debate, dado que los participantes procedían de diferentes realidades, de formaciones y de historias totalmente distintas. El texto de lo que podría constituir la introducción propia del Libro Blanco seria sucesivamente cambiado y matizado cada vez que se le hacía una nueva lectura.

En aquellos momentos, todavía eran pocas las personas que participaban en el grupo de debate. Algunos ya venían en representación de determinados colectivos de profesionales más o manos organizados en Asociaciones. Otros asistían a título personal porque el tema simplemente les interesaba. Únicamente Valencia, Murcia, Cataluña y Galicia tenían creada, entonces, una asociación de profesionales de Atención Temprana. El tipo de formación era muy variada, dependiendo de la disciplina a la cual se dedicaban, de esta manera pudimos trabajar sin demasiados conflictos, algunos médicos rehabilitadores, neuropediatras, neonatólogos, psicólogos, pedagogos, profesores de universidad, etc.

Fue a partir de sucesivas reuniones y de poner en común experiencias y reflexiones de representantes de las comunidades autónomas que contaban con organismos aglutinadores de profesionales que trabajaban por el mismo tema -en este caso, en servicios, hospitales $\mathrm{u}$ universidades- que se fueron animando a participar otros grupos asociados.

Poco a poco, este grupo -ya numeroso- se fue cohesionando, y era constante en los encuentros que se iban realizando -aproximadamente cada mes y medio-. El sistema seguido para la redacción era el siguiente: se trabajaba en forma de comisiones, las cuales iban elaborando y redactando cada uno de los apartados que tendría que tener el futuro Libro Blanco. El día de la reunión general, se daba lectura a cada uno de los textos elaborados, para luego discutir, cambiar o ampliar los conceptos utilizados. Ello permitía llegar a un consenso bastante general entre todos los participantes en las reuniones.

Cada vez más, se iba percibiendo que este documento tendría que establecer las bases del futuro de la Atención Temprana en nuestro país, definir unos derechos aplicables a todos los niños y niñas y establecer unos criterios sólidos en cuanto a la organización y a los recursos en los diferentes niveles de acción: la prevención, la detección, el diagnóstico y la intervención en la población infantil que lo requiera, en su familia y en su entorno.

Las reuniones de trabajo se iban realizando en una ciudad diferente cada vez. Los profesionales que trabajaban en aquella localidad procuraban el espacio de encuentro. Finalmente, y cuando ya éramos muchos los implicados en esta tarea, decidimos realizar las reuniones en Madrid, ya que era el lugar equidistante para todos. Esta decisión y la necesidad de poder obtener un soporte institucional, fue la que nos decidió a ponernos en contacto con el Real Patronato sobre Discapacidad (en adelante, RPD).

En noviembre de I997, se pidió una entrevista con el objetivo de presentar el trabajo que se estaba realizando y sus objetivos. Podemos afirmar que éste fue un momento muy importante para nuestro grupo y nuestro propósito. Demetrio Casado -entonces director técnico- nos recibió y acogió en nombre del Real Patronato con gran generosidad y, sobre todo, reconociendo todo el trabajo que se estaba realizando.

En febrero de I998, se convocó la primera reunión en la sede del RPD. Se nos ofreció la sala de reuniones de la institución. La gran mesa ovalada, los amplios sillones giratorios, el micrófono ante cada uno de nosotros, que no utilizamos jamás, las botellas de agua para cada asistente... nos hicieron sentir, al comienzo, un tanto cohibidos. Poco a poco empezamos a sentirnos en el RPD como en nuestra casa común. 
Entonces trabajábamos por la mañana y la tarde de los viernes. Cuando se decidía continuar el sábado por la mañana -hecho muy frecuente-, el departamento de Neonatología del Hospital San Carlos a través de Arizcun o el departamento de Pedagogía de la Universidad Complutense, a través de Pilar Gutiez, nos aportaban un espacio para podernos reunir y seguir trabajando.

En la reunión de febrero de I998, se presentó al RPD un proyecto escrito sobre los objetivos y el contenido que tendría que tener el "Libro Blanco" con la finalidad de poder conseguir alguna subvención económica (los gastos de viajes y estancia en Madrid de las personas que -de forma absolutamente voluntaria- fueron participando en el trabajo se cubrían gracias a las aportaciones de las diferentes asociaciones a las que cada uno pertenecía).

A medida que iba avanzando la elaboración del documento, se iban incorporando nuevas personas. Profesionales provenientes de diferentes ámbitos y disciplinas: Mercedes del Valle (psicóloga del Departamento de Neonatología del Hospital San Carlos), Maite Andreu (profesora de Psicología de la Universidad Complutense de Madrid), Eugenia Lara (psicóloga de Almería), José Cardama, de Galicia, Inmaculada Ramos y $\mathrm{M}^{\mathrm{a}}$ Antonia Márquez (Neuropediatra y psicóloga del Hospital Virgen de la Macarena de Sevilla), Carmen Linares (profesora de la Universidad de Málaga), Ma Luisa Poc (neuropediatra de la Rioja), Julio Pérez de la Asociación de Murcia y Miguel Ángel Rubert, que como gerente de la Associació Catalana d'Atenció Precoç, se incorporó para asesorarnos en algunos temas administrativos y de organización. También en esta reunión se establece la necesidad de una secretaria que pueda elaborar las actas, realizar las convocatorias y en el caso de que se pudiera conseguir alguna ayuda económica, buscar formas de distribución. Este trabajo de secretaria lo asumió Fátima Pegenaute.

Fue aproximadamente a partir de este encuentro con el RPD que el grupo fue tomando mayor conciencia de su cohesión y aportación y pudo tomar un nombre. Se autodenominó Grupo de Atención Temprana (GAT). El anagrama que lo identificó a partir de aquel momento, lo aportó la Asociación de AT de la Comunidad Valenciana. El nombre que nos identificaba como un grupo de trabajo, GAT, con el tiempo y la creación de más asociaciones, se trasformó en Federación Estatal de Asociaciones de Profesionales de la Atención Temprana. No obstante, nunca quisimos que desapareciera el nombre que, por primera vez, nos dio identidad e hizo que se nos conociera en todos los ámbitos de la primera infancia.

En la reunión celebrada en mayo del I998, se dio un giro importante en lo que serian los contenidos del Libro Blanco. Si hasta el momento el tema central había sido la Atención Temprana -entendida como intervención que se debería dar exclusivamente, desde los servicios de tratamiento (CDIAP) - a partir de esta fecha, se decidió ampliar el concepto y se asumió que cuando hiciéramos referencia a la AT se debería incluir también otros servicios, como los que tratan la prevención, el cuidado y la atención a los niños y a sus familias desde otros ámbitos, como los Servicios de Atención Primaria, las escuelas infantiles, los servicios de neonatología y neurología, etc.

Conforme el grupo iba trabajando y avanzando sobre el tema, se vio necesario iniciar las primeras presentaciones del proyecto. Creíamos que era importante que las diversas instituciones públicas o privadas, tanto a nivel estatal como autonómico, pudieran estar informadas y pudieran ofrecer su apoyo institucional. Por aquellas fechas, FEAPS a través de sus comisiones estaba elaborando un proyecto con ciertos elementos comunes -Manual de Buenas Prácticas en Atención Temprana- en el que participaban algunas profesionales del GAT.

Fue por entonces que se incorporaron a la lista de participantes del GAT Pilar Gutiez, profesora de Pedagogía de la Universidad Complutense de Madrid; Xavier Tapias y Carmen Manjón, psicólogos pertenecientes al ámbito de la salud mental del País Vasco; José Cardama de la Asociación de Galicia; Carmen Narváez y Pilar Bedia, psicólogas de la Asociación AMICA 
de Cantabria, y Manuel Tejero, psicólogo de Toledo, de la Asociación de Castilla-La Mancha.

Poco a poco, el Libro Blanco iba tomando forma y volumen. Los debates se hacían cada vez mas difíciles de gestionar debido al número de participantes cada vez más elevado, dado el interés que el objetivo suscitaba. Se decidió, finalmente, crear diferentes comisiones, en torno a cada uno de los temas tratados, que se reunieran antes de cada encuentro general y pudieran traer enmiendas y aportaciones concretas.

En las reuniones del GAT, además de trabajar en el documento del Libro Blanco, se trataron otros asuntos, como la formación de nuevas asociaciones y la manera de constituir y formalizar legalmente el grupo. Ello permitiría en el futuro garantizar tanto su continuidad como su representatividad en los foros relacionados con el tema que nos afectaba.

Con la entrada del año I999, por primera vez el GAT recibió la primera ayuda económica a través del Real Patronato sobre Discapacidad. GENYSI y ACAP como instituciones con poder jurídico se constituyeron como organismos asesores en los aspectos de índole económica. También en este año, concretamente en noviembre, en una entrevista con Demetrio Casado, el RPD se hizo responsable de publicar, distribuir a nivel estatal y presentar de forma oficial el Libro Blanco una vez se hubiera concluido.

Finalmente, y después de haber pasado una revisión literaria, realizada por Elvira Villalobos, del Centro Español de Documentación sobre Discapacidad, el Libro Blanco fue presentado oficialmente el día I 6 de junio del 2000 en Madrid. El acto fue presidido por el secretario general del RPD, acompañado por representantes de diversos ministerios. Por parte del GAT, intervino Fátima Pegenaute. La sala estaba llena y creo que fue un momento muy emotivo para todos.

Habíamos llegado al final de un camino, largo y difícil pero lleno de buenos paisajes. Un camino que para todos los que participamos y nos implicamos fue una experiencia muy rica no sólo a nivel científico sino también a nivel humano.

Nos había ofrecido la oportunidad de escuchar, de discutir a partir de diferentes lenguajes, tolerar diferencias y encajar diferentes realidades $\mathrm{y}$ diferentes formas de ver las cosas.

A esta primera presentación le seguirían muchas más en diferentes comunidades autónomas. La expectación y el interés suscitados por este documento fueron grandes, de forma que a los cuatro meses después de su primera presentación, ya se habían agotado los I 500 ejemplares de la primera edición. Hubo que hacer reediciones. En 2002, se habían distribuido 7000 ejemplares, no sólo en nuestro país sino también en el extranjero.

Estas presentaciones, en general, se daban en el contexto de algunas Jornadas organizadas por diferentes instituciones y se hacían coincidir con la concesión del Premio Reina Sofía, otorgado también por el Real Patronato sobre Discapacidad. El año 2000 estuvo relacionado también con la primera infancia: "Apoyo al desarrollo de los niños nacidos demasiado pequeños y demasiado pronto“, de Carmen R. Pallás, Javier de la Cruz y M M Carmen Medina. El Premio facilitaba una conexión entre el ámbito médico y nuestro trabajo de prevención y cuidados tempranos.

\subsubsection{Aportaciones del Libro Blanco}

El Libro Blanco había nacido con el deseo de constituirse como un referente para todos los profesionales de los ámbitos en los que se atiende a los niños en las primeras etapas, para las administraciones públicas del país, para las entidades asociativas y, evidentemente, para las familias de todos aquellos niños susceptibles de ser atendidos.

Una de las aportaciones destacables del Libro Blanco es entender el desarrollo infantil como un proceso dinámico, sumamente complejo, fruto de la interacción entre factores genéticos, factores ambientales y factores sociales. De 
lo que se deduce que el abordaje en Atención Temprana ha de ser global, y que los equipos de atención han de estar formados por profesionales de los diferentes ámbitos (médico, psico-social y educativo). Estos profesionales deben trabajar de forma coordinada e interdisciplinar.

La AT, por consiguiente, no podía ser vista exclusivamente como una vertiente de la rehabilitación, de la intervención psico-social o de la educación, sino que debía formar parte de un proceso integral que tuviera como objetivo único el desarrollo armónico de los niños, integrados en un contexto socio-familiar y cultural.

El Libro Blanco defiende un modelo de atención global e interdisciplinar que tenga en cuenta en todo momento y desde cualquier ámbito, las características evolutivas y las necesidades de cada niño, en todos los aspectos, sus capacidades y sus dificultades, su historia y su proceso evolutivo, las características y las necesidades de la familia y los recursos de su entorno más inmediato.

En el nuevo documento se valora la necesidad de crear y coordinar programas de detección, en población de alto riesgo biológico y social y en programas de diagnóstico temprano de cualquier tipo de alteración o trastorno en el desarrollo, con la finalidad de poder iniciar en los casos pertinentes una intervención adecuada y cuanto antes a las necesidades que presente el niño y su familia.

Se señala la importancia de crear canales de coordinación que posibiliten una planificación eficaz y que sirva de cauce para la transferencia de la información. Canales a nivel interinstitucional e intrainstitucional que abarquen desde los ministerios implicados, las consejerías de las comunidades autónomas y las administraciones locales, a las organizaciones de usuarios y a los propios profesionales que tienen a su cargo la atención del niño. Solamente con esa fórmula se evitarán pasos incoherentes y duplicados entre todos los implicados en el proceso.
Se amplía el tipo de población diana a la cual ofrecer este tipo de intervención. Ya no se contempla de forma exclusiva a los niños con discapacidad sino que se amplía a cualquier niño que presente o pueda presentar alguna dificultad o trastorno que pueda afectar a su desarrollo. La inclusión del término riesgo, no únicamente entendiéndolo a nivel biológico sino también en los aspectos sociales, marcó un hito importante en el tema de la prevención.

Apoya el concepto de sectorización como algo necesario para acercar el servicio a las familias y posibilitar una mayor y mejor coordinación entre otros servicios que también prestan atención al niño y a su entorno durante esas primeras etapas (servicios de atención a la salud, servicios sociales y centros educativos y de ocio). La gratuidad y la universalidad del acceso y las diferentes oportunidades que los servicios ofrecen, se considera un derecho para todos los niños y sus familias sin distinción de dónde vivan o dónde hayan nacido.

Favorece el que a partir de su publicación los centros o equipos de Atención Temprana -llamados así hasta el momento- tomen el nombre de Centros de Desarrollo Infantil y Atención Temprana (CDIAP). Según este modelo, el CDIAP asume las funciones de acogida, evaluación diagnóstica, intervención terapéutica, seguimiento de los niños con riesgo biológico o social y coordinación con los diferentes ámbitos del territorio que también se ocupan de estos niños y sus familias.

Finalmente, conviene recordar que del Libro Blanco se han realizado diversas ediciones en papel y ha sido citado en innumerables artículos, libros y conferencias. También se han realizado varias traducciones del documento:

- Libro Blanco da Atención Tempera. Traducido al gallego en el año 200 I con la colaboración del Ministerio de Trabajo y Asuntos Sociales y la Asociación Galega de Atención Temperá.

- Llibre Blanc de l'Atenció Precoç. Traducido al catalán y editado por la Diputació de Barcelona en el año 200I. 
- White Paper on Early Intervention. Traducido al inglés por la Asociación Madrileña de la Atención Temprana (AMPAT) y editado por el RPD en el año 2005.

- En árabe, traducido por la Asociación Hanna, de Tetuán, y editado por el RPD.

- El Libro Blanco fue traducido al lenguaje Braille en el año 200 I y editado en forma auditiva (casette) por la Organización Nacional de Ciegos de España (ONCE).

\subsection{La Federación de Asociaciones de Profesionales}

Una vez presentado el Libro Blanco, gran parte de las personas que componían el GAT estuvieron de acuerdo en que se había abierto un camino por explorar y por el que seguir marcando objetivos y trabajando de forma conjunta. Además, durante todo aquel tiempo, habían nacido nuevas asociaciones en las diferentes autonomías, que fueron incorporándose al grupo ya existente y enriqueciendo la participación y la representación de todos los profesionales.

Así, en fecha 26 y 27 de enero del 200I, con la participación de ocho asociaciones formalizadas (Andalucía, Aragón, Castilla-La Mancha, Cataluña, Extremadura, Galicia, Murcia y Valencia) se constituyó la Federación Estatal de Asociaciones de Profesionales de la Atención Temprana. Se eligió como primera presidenta a Gracia Milla, representante de la Asociación de Valencia. La junta se constituyó con Francisco Alberto García -de la Asociación de Murcia- como vicepresidente, Fátima Pegenaute -representante de la Asociación de Cataluña-, que asumió la secretaría, y Carmen Linares -representante de Andalucía- que asumió la tesorería.

Los objetivos más destacados de la Federación:

- Organizar una entidad estable de representación de los profesionales de la AT de las diferentes comunidades autónomas.

- Promocionar la AT y desarrollar proyectos comunes, tendentes a mejorar la calidad asistencial y el fomento de su desarrollo científico.

- Articular una representación estable de la AT española en los diversos foros europeos e internacionales.

La metodología de trabajo de este grupo se ha caracterizado por ser muy abierta a las aportaciones de todos los participantes y por el debate constructivo y flexible que permitía alcanzar consensos. Aunar diferentes perspectivas desde diversas disciplinas y servicios dependientes de distintas titularidades era uno de los mayores retos con los que nos encontramos. La Atención Temprana, en sus primeros pasos, requería tomar muchas decisiones, definir marcos teóricos, delimitar ámbitos de actuación, distribuir funciones y para ello era imprescindible un alto grado de flexibilidad. El grupo siempre ha estado abierto a acoger profesionales procedentes de distintas comunidades y disciplinas y los profesionales que han formado parte del GAT han mostrado un grado de implicación en el trabajo y de vocación por la Atención Temprana.

\subsection{Otros documentos del GAT}

Una vez terminado y presentado el Libro Blanco, el GAT se propuso llevar adelante la elaboración de algunos trípticos divulgativos dirigidos a diferentes ámbitos (familias, pediatras, educadores, etc.), con el objetivo de dar a conocer los conceptos básicos y de más relevancia del Libro Blanco y hacerlos llegar a cuantos profesionales y entidades estuvieran interesadas en la materia.

\subsubsection{Organización Diagnóstica para la Atención Temprana (ODAT)}

Uno de los temas que preocupaba a los miembros del GAT y que surgía muy frecuentemente en las reuniones de la Federación era el tema del diagnóstico y las diferentes clasificaciones diagnósticas utilizadas por los profesionales en los Centros de Desarrollo Infantil y Atención Temprana. 
Los profesionales de la AT hemos considerado que, de cara a las familias y también a los propios profesionales responsables de la intervención -sea ésta de la índole que fuere-, el diagnóstico funcional, caracterizado por destacar las capacidades y las dificultades que presenta el niño, era el más idóneo para comprender las necesidades de éste y organizar el tipo de intervención y los recursos que pudiera necesitar. De igual manera, creíamos que era interesante poder utilizar un lenguaje común, de cara a realizar estudios epidemiológicos y proyectos de investigación.

Cuando el GAT empezó a debatir acerca de la clasificación diagnóstica, las clasificaciones más utilizadas en el ámbito de la AT eran las internacionales: el DSM IV y V y la Clasificación Médica de las Enfermedades (CIE ro). También, y sobre todo en el ámbito de la discapacidad, en los centros de valoración se valoraba a los niños utilizando la Clasificación CIF de la Organización Mundial de la Salud (OMS) sobre discapacidad.

Era generalizado el sentir de los profesionales en cuanto a la dificultad que suponía encontrar la formulación precisa para designar las dificultades que los niños podían presentar en estas primeras etapas, sobre todo cuando se trataba de trastornos más ligados a los aspectos emocionales que a los médicos.

En alguna autonomía, como Cataluña, se estaba imponiendo en ese momento, la utilización de la Clasificación Diagnóstica o a 3 (Diagnostic Classification of Mental Health and Developmental Disorders of Infancy and Early Childhood) para este tipo de casos, completándose con la utilización del CIE ro para aquellos casos en donde el diagnóstico podía tener un origen y unas características médicas.

La decisión unánime del GAT fue la de crear una organización diagnóstica nueva y particular que asumiera todas las categorías diagnósticas comprendidas en las otras clasificaciones y que tuvieran relación con los niños en estas primeras etapas de la infancia.
A esta organización se le llamó Organización Diagnóstica para Atención Temprana (ODAT) (GAT, 2004). Se editó en el año 2004 y el coordinador principal del trabajo realizado fue Juan Carlos Belda junto con José Cardama. Una vez editada se llevó a cabo un trabajo de validación entre algunos CDIAP/s del país y a partir de los resultados se realizó una actualización en el 2008.

Este documento fue traducido al portugués en el año 2009 .

\subsubsection{Recomendaciones técnicas para el desarrollo de la Atención Temprana}

Este documento (GAT, 2005) constituyó un complemento muy eficaz que surgió cinco años después de la edición del Libro Blanco. Su realización fue liderada por Jaime Ponte y tuvo como finalidad profundizar en aspectos esenciales relacionados con los objetivos y los procedimientos de intervención, ofreciendo un conjunto de recomendaciones seleccionadas por su impacto, para el desarrollo infantil y la AT.

\subsubsection{Revisión y nuevo diseño del calendario del desarrollo}

Nos referimos a un desplegable divulgativo -calendario del desarrollo del niño de o a I 8 meses-destinado a familias y profesionales que atienden a niños, mostrando los grandes hitos de la evolución infantil.

El impreso, que se había utilizado y distribuido en Madrid a todas las familias cuando iban a tener un hijo, hasta ese momento era la traducción al castellano del que años atrás se había creado con el soporte del Ajuntament de Barcelona, y en el que habían participado Concha Bugié y $\mathrm{M}^{\mathrm{a}}$ Teresa Folguera.

Los miembros del GAT consideraron conveniente adecuar no el contenido sino sólo los dibujos del calendario a las circunstancias actuales. Se trataba de reflejar en las imágenes aspectos importantes, como la diversidad de la 
realidad social y la participación activa de los padres varones en el cuidado y la crianza de los hijos. Anna Maldonado diseñó los nuevos dibujos. Fue editado por el RPD y distribuido por todas las comunidades autónomas. Ha sido traducido al árabe por la Asociación Hanna de Tetuán (Marruecos) en el año 20 Io.

\subsubsection{Guía del desarrollo infantil desde el nacimiento hasta los 6 años}

Una vez reeditado el calendario, valoramos que, si bien éste podía y de hecho había sido un instrumento adecuado para proporcionar a los padres unos referentes sobre el desarrollo del niño y alertarles de cualquier desviación en ese desarrollo, podía ser también de gran interés explicarles en cada etapa cuáles eran las principales necesidades de su hijo y cómo ellos podían comprenderlas mejor y responder de la forma más adecuada.

Así como el calendario comprende la etapa de o a los I 8 meses, la guía se alarga hasta los 6 años. Cada periodo está organizado en tres apartados: las características principales del niño, sus necesidades y lo que pueden hacer los padres en respuesta a esas necesidades.

Este trabajo fue coordinado por Sonsoles Perpiñán, Emilio J. García y Fátima Pegenaute y editado por el RPD en el año 20I0 (GAT, 20Iо). En su elaboración también participaron Julio Pérez de Murcia y Cristina Cristóbal que en aquel momento ejercía el cargo de presidenta de l'Associació Catalana d'Atenció Precoç.

\subsubsection{La primera noticia}

Con este título (GAT, 20I I) se dio a conocer un estudio acerca de los procedimientos profesionales, las vivencias y las necesidades de los padres cuando se les informa de que su hijo/a tiene una discapacidad o un trastorno en el desarrollo. Requirió, por un lado, realizar una revisión de la documentación científica y literaria existente y relacionada con el tema y, por otro, un estudio estadístico y vivencial que se llevó a cabo con la generosa aportación de muchas familias, pertenecientes a diferentes asociaciones de afectados.

Estas familias dieron respuesta a un cuestionario en el que se incluían preguntas sobre el cómo, el dónde y el cuándo se les transmitió la comunicación acerca de la patología que sufría o podía sufrir su hijo, recogiéndose también en el mismo las diferentes vivencias emocionales que habían tenido en aquel momento.

El documento, realizado a partir del repaso bibliográfico y de los resultados obtenidos en el estudio, finalizó con una serie de recomendaciones dirigidas a los profesionales que trabajan en el campo de la infancia y que tienen que enfrentarse en muchas ocasiones a dar esta "primera noticia" a una familia.

Editado por el Real Patronato sobre Discapacidad en el año 20 I I, fue un trabajo realizado sobre la elaboración previa de una comisión de trabajo que coordinaron Jaime Ponte y Sonsoles Perpiñán.

\subsubsection{Estudio sobre la realidad de la Atención Temprana en nuestro país}

Pasados siete años de la edición del Libro Blanco, se pensó en la idoneidad de realizar un estudio completo, en el ámbito nacional, sobre la realidad que en estos momentos tenia la Atención Temprana en nuestro país. Este trabajo (GAT, 20I I) supuso un análisis en profundidad de las características de la población atendida y de los modelos de trabajo de los diferentes CDIAP/s que habían sido creados en las diferentes comunidades autónomas.

En el trabajo se planteaban los siguientes objetivos:

- Conocer la legislación concreta en materia de Atención Temprana de cada comunidad autónoma.

- Determinar el número de servicios implicados en actividades de Atención Temprana y su dependencia, dentro de los tres ámbitos: social, educativo y sanitario. 
- Concretar los objetivos específicos en materia de AT de cada uno de estos servicios y la forma de organización.

- Determinar los medios y recursos disponibles a fin de poder cumplimentar los objetivos propuestos.

- Determinar la relación existente entre el servicio de AT y los diferentes servicios implicados en la atención al niño y a la familia.

- Conocer el circuito por el que pasa el niño desde que se detecta un factor de riesgo que necesita de un seguimiento o desde que se detecta un trastorno en el desarrollo.

Este trabajo fue llevado a cabo gracias a la coordinación que protagonizó la Asociación de Atención Temprana de Aragón, sobre todo en las personas de Pilar López, Raquel Cabrerizo y Lourdes Navarro. Fue editado en forma digital por el RPD y presentado en numerosas Jornadas.

\subsection{Otras actividades}

Año tras año, a partir del crecimiento de servicios de AT en los diferentes territorios, se fueron creando asociaciones de profesionales. Y, a su vez, éstas se iban integrando en la Federación. De esta forma, se plantearon nuevos objetivos y actuaciones.

\subsubsection{Jornadas de formación e intercambio}

Como ya hemos comentado, antes de constituirnos como Federación, el GAT habíamos participado en las Jornadas Interdisciplinares que organizaba GENYSI. Esas Jornadas se realizaban en Madrid y tenían un público asiduo dado que su nivel científico era excelente.

Hacia el 2006, una vez creada la Federación estatal nos acogimos a la posibilidad de organizar de forma autónoma, nuestras propias Jornadas anuales. Se celebrarían en una comunidad autónoma distinta cada vez y tendrían como objetivos la formación y reflexión de los profesionales y el intercambio de experiencias. No se ocultaba el interés de la Federación por dar a conocer a los responsables políticos y a la población la realidad y la importancia de la AT en la pronta atención de los niños con discapacidades. Las primeras Jornadas se realizaron en Mérida (Extremadura) en 2006. La organización era responsabilidad de la Asociación propia de la comunidad autónoma y se realizaban con el soporte institucional y económico de las Administraciones de cada autonomía y del RPD.

En años sucesivos, fueron celebrándose:

- $\quad$ En 2007, en Toledo.

- En 2008, en Zaragoza.

- En 2009, en Murcia.

- En 2oro, en Palma de Mallorca.

- En 2or I, en Sevilla.

- En 20r2, en Valencia.

- En 20r4, en Santiago de Compostela.

- $\quad$ En 2015 , en Elche.

A la vez y ya desde la creación del grupo GAT, esta institución era invitada frecuentemente por otras organizaciones del país a participar en numerosas Jornadas organizadas desde diferentes instituciones y ámbitos. El objetivo siempre era, o presentar los trabajos y documentos que se iban realizando, o debatir sobre temas relacionados con la AT en general. Así, la Federación estuvo presente en las Jornadas de ASPRODIC o en las de la Asociación de Acondroplasia (2007), en la organizadas por el Servei de Neonatología del Hospital Vall d'Hebron, sobre AT y prematuridad y en las organizadas por la Asociación Síndrome de Down en Mallorca (2008), en las organizadas por el Intitut Català d'Assistència y Serveis Socials de Cataluña (20IO), etc.

\subsubsection{Premios de investigación y premios honoríficos}

La Federación, a partir del año 2009, decidió crear unos premios a la investigación en la AT, con la intención de incentivar el interés de 
los profesionales de la AT por este aspecto, y unos premios honoríficos con el objetivo de valorar públicamente la trayectoria de algunos profesionales que se habían destacado por su trabajo y aportaciones a la AT.

Para poder materializar estos premios en ayudas económicas, sobre todo el de investigación, se acudió, a través de Rosa Mayo, a la Fundación de la Organización Nacional de Ciegos de España (ONCE). De hecho, la colaboración con dicha Fundación siempre ha sido muy fluida y positiva, facilitando espacios al GAT para la celebración de algunas reuniones.

Los premios honoríficos, hasta el momento, se han otorgado a Carmen Linares en 2009, a $\mathrm{M}^{\mathrm{a}}$ Gracia Milla en 20 Io y a Concha Bugié en 2014 .

\subsubsection{Función representativa}

La Federación siempre se ha considerado como una plataforma de representación de todos los profesionales de la AT del país. Su objetivo es mejorar la calidad asistencial en el conjunto del territorio y articular una representación estable en los diferentes foros nacionales e internacionales. De esta manera, ha sido tenida en cuenta cada vez más por otros organismos e instituciones autonómicas y estatales, con el fin de colaborar y participar en diferentes eventos.

En el año 2003, la revista Minusval (Minusval, 2003) realizó un encargo a la presidenta de la Federación, en aquel momento $\mathrm{M}^{\mathrm{a}}$ Gracia Milla, con el fin de editar un número dedicado a la AT. En él colaboraron algunos de los profesionales que en aquel momento participaban también en la Federación estatal. Años más tarde, en el 2006 (Minusval, 2006), la misma revista realizó y editó una entrevista a la misma presidenta con el fin de poder informar a los lectores sobre la realidad de la AT en nuestro país.

En el año 2007 y gracias a la colaboración de Carmen Narváez y Pilar Bedia, de Cantabria, se inició la creación de la web de la Federación, que supondrá más adelante que la información generada por el GAT y los documentos creados a través de los años puedan llegar a más personas e instituciones no solamente a nivel estatal sino también a nivel internacional.

El RPD pidió nuestra participación en el jurado para la concesión del Premio Reina Sofía del año 2008.

A lo largo de estos años se mantuvieron también algunos contactos con el Comité Español de Representantes de Personas con Discapacidad (CERMI), con la Federación de Asociaciones a favor de Personas con Discapacidad Intelectual (FEAPS, ahora Plena Inclusión España), con la Asociación Estatal de Personas con Acondroplasia... siempre con la finalidad de aunar esfuerzos y hacer más fácil la atención a todos aquellos niños y a todas las familias que pasaban y tendrían que pasar en un futuro por nuestros centros de Atención Temprana.

\section{Para terminar}

Seguramente en el camino del recuerdo, que siempre es frágil, he olvidado a muchas personas $\mathrm{u}$ organismos, acontecimientos o actividades que durante todos estos años han tenido importancia y han pasado y colaborado en un momento u otro con el GAT. Personas que nos dieron lo mejor de sí mismas -en el sentido humano y profesional- que dejaron su huella, que ofrecieron su experiencia y su saber, que nos ayudaron a debatir y pensar. Debemos reconocer que la memoria es siempre selectiva $-\mathrm{y}$ a veces, traidora-, además de que los recuerdos también son siempre subjetivos y poseen una carga emotiva importante. Desde estas líneas, deseo invitar a otros participantes a aportar su visión del proceso o a completar o corregir datos que, sin duda, contribuirán a mejorar y objetivar el relato.

Este artículo pretendía, por una parte, recoger y sistematizar una serie de vivencias y de hechos que se han ido sucediendo a través de todos 
estos años en torno a la AT y de los cuales creo que muchas personas se sentirán protagonistas activos. Por otra, reconocer los grandes progresos realizados por nuestro país en AT, de los cuales se han beneficiado muchísimos niños y niñas y sus respectivas familias. Finalmente, como ya se anunció al comienzo, quería rendir un homenaje de reconocimiento y gratitud a las instituciones y a todos y todas cuantos participaron en esta grata aventura.

\section{Referencias bibliográficas}

GAT (20I I): La Atención Temprana en España, Madrid: Real Patronato sobre Discapacidad.

GAT (20I I): La primera Noticia. Estudio sobre los procedimientos profesionales, las vivencias y las necesidades de los padres cuando se les informa de que su hijo tiene una discapacidad o trastorno en el desarrollo, Madrid: Real Patronato sobre Discapacidad.

GAT (2010): Guía del Desarrollo infantil desde el nacimiento hasta los 6 años, Madrid: Real Patronato sobre Discapacidad.

GAT (2005): Recomendaciones Técnicas para el Desarrollo de la Atención Temprana, Madrid: Real Patronato sobre Discapacidad.
GAT (2004): Organización Diagnóstica para la Atención Temprana (ODAT), Madrid: Real Patronato sobre Discapacidad.

GAT (2000): Libro Blanco de la Atención Temprana, Madrid: Real Patronato de Prevención y Atención a Personas con Minusvalía.

VV.AA. (2006): "La Atención Temprana”. Minusval, I 59: I2-I 5.

VV.AA. (2003): “La Atención Temprana”. Minusval, 3 . 